\title{
Macular edema after cataract surgery in eyes with and without pseudoexfoliation syndrome
}

\section{Ilveskoski, Lotta}

2019-09

Ilveskoski , L, Taipale , C , Holmstrom , E J \& Tuuminen, R 2019 , ' Macular edema after cataract surgery in eyes with and without pseudoexfoliation syndrome ' , European Journal of Ophthalmology , vol. 29 , no. 5 , pp. 504-509 . https://doi.org/10.1177/1120672118799622

http://hdl.handle.net/10138/320955

https://doi.org/10.1177/1120672118799622

publishedVersion

Downloaded from Helda, University of Helsinki institutional repository.

This is an electronic reprint of the original article.

This reprint may differ from the original in pagination and typographic detail.

Please cite the original version. 


\title{
Macular edema after cataract surgery in eyes with and without pseudoexfoliation syndrome
}

European Journal of Ophthalmology 2019, Vol. 29(5) 504-509

(C) The Author(s) 2018

Article reuse guidelines:

sagepub.com/journals-permissions DOI: 10.1 | $77 /$ | | 206721 I8799622 journals.sagepub.com/home/ejo

(\$) SAGE

\author{
Lotta Ilveskoski 1,2, Claudia Taipale ${ }^{1,2}$, Emil J Holmström ${ }^{1,3}$ \\ and Raimo Tuuminen ${ }^{1,4}$
}

\begin{abstract}
Background: The purpose of the study was to identify macular edema after cataract surgery in eyes with and without pseudoexfoliation syndrome. The study was a post-hoc analysis of a randomized, double-blind, prospective single-center study. Patients were enrolled between January 2016 and October 2016 as per the national guidelines for the management of cataract in the Department of Ophthalmology, Kymenlaakso Central Hospital, Kotka, Finland.

Methods: One hundred and fifty-six eyes of 149 patients undergoing routine cataract surgery. Postoperatively antiinflammatory medication was either dexamethasone $(N=78)$ or diclofenac $(N=78)$. Spectral domain optical coherence tomography imaging and laser flare meter measurement of the anterior chamber were conducted before surgery and at the control visit 28 days postoperatively.

Results: Baseline variables were comparable between eyes with pseudoexfoliation syndrome $(N=32)$ and those without $(N=124)$, except for intraocular pressure $(P=0.002)$ and glaucoma medication $(P<0.00 \mathrm{I})$. In patients having pseudoexfoliation syndrome, central retinal thickness increase (mean \pm standard error of the mean) was $63.3 \pm 35.5 \mu \mathrm{m}$ for dexamethasone and $17.6 \pm 5.8 \mu \mathrm{m}$ for diclofenac, compared to $28.9 \pm 8.0 \mu \mathrm{m}(P=\mathrm{NS})$ and $6.9 \pm 1.3 \mu \mathrm{m}(P=0.014)$ in eyes without pseudoexfoliation syndrome, respectively. Aqueous flare at 28 days was $25.8 \pm 5.4 \mathrm{pu} / \mathrm{ms}$ for patients with pseudoexfoliation syndrome and $18.3 \pm 1.8 \mathrm{pu} / \mathrm{ms}$ for those without $(P=0.030)$. Best corrected visual acuity gain and best corrected visual acuity at 28 days were less in patients having pseudoexfoliation syndrome compared to those without $(0.39 \pm 0.07$ vs $0.59 \pm 0.03$ decimals, $P=0.007$; and $0.77 \pm 0.06$ vs $0.92 \pm 0.03$ decimals, $P=0.008$, respectively).

Conclusion: Eyes with pseudoexfoliation syndrome may be predisposed to an increased aqueous flare and macular edema after cataract surgery. This study outlines the need to determine the optimal anti-inflammatory medication after cataract surgery in patients with pseudoexfoliation syndrome.
\end{abstract}

\section{Keywords}

Aqueous flare, cataract, central retinal thickness, macular edema, phacoemulsification, pseudoexfoliation syndrome

Date received: 22 April 2018; accepted: 20 August 2018

\section{Introduction}

Cataract surgery is one of the most common surgical procedure, and yet the amount of surgeries is expected to rise within next decades. ${ }^{1}$ With modern phacoemulsification techniques, cataract surgery is safer than ever. However, the procedure is not risk-free. With the high number of operations, complications are occasionally seen. Pseudophakic cystoid macular edema (PCME; IrvineGass syndrome), impairing visual acuity gain, is the most common postoperative complication of cataract surgery.
'Helsinki Retina Research Group, University of Helsinki, Helsinki, Finland

2Department of Ophthalmology, Helsinki University Hospital, Helsinki, Finland

${ }^{3}$ Transplantation Laboratory, University of Helsinki, Helsinki, Finland

${ }^{4}$ Unit of Ophthalmology, Kymenlaakso Central Hospital, Kotka, Finland

Corresponding author:

Raimo Tuuminen, Unit of Ophthalmology, Kymenlaakso Central Hospital, Kotkantie 4I, FI-482 I0 Kotka, Finland.

Email: raimo.tuuminen@helsinki.fi 
The incidence of PCME is about $1 \%$ among patients with no known risk factors. Studies have identified diabetes, uveitis, presence of posterior segment comorbidity (epiretinal membrane/macular pucker, retinal vein occlusion, retinal detachment) and perioperative complications (capsule rupture) as risk factors for PCME. 2,3

Pseudoexfoliation syndrome (PXF) was discovered 100 years ago by a Finnish ophthalmologist, John G. Lindberg. PXF is an age-related disorder that associates with cataract development and is the most common cause of secondary open-angle glaucoma. ${ }^{4,5}$ PXF is caused by fibrillar-granular extracellular material which gradually deposits in the anterior segment of the eye, and other body tissues. PXF exists worldwide affecting with estimation over 60 million people. ${ }^{6,7}$ The prevalence of PXF in the Scandinavian population is exceptionally high, where the mean prevalence of PXF for individuals aged over 60 years is above $20 \%$ and increases significantly with age. ${ }^{8}$

PXF is identified as a peri- and postoperative risk of cataract surgery. Weak structures predispose eyes to greater incidence of zonular dialysis. Moreover, PXF associated with blood-aqueous barrier disruption after intraocular surgery. ${ }^{9}$ Eyes with PXF were reported to have extensive and prolonged aqueous flare following uneventful cataract surgery. The fragility of the bloodaqueous barrier represented by increased flare after intraocular operations is suspected to predispose PXF eyes to macular edema. ${ }^{10}$

To prevent PCME, topical nonsteroidal anti-inflammatory drug (NSAID) eye drops have been recommended after routine cataract surgery. ${ }^{11,12}$ Aggressive anti-inflammatory medications including a combination of steroids and NSAID treatment have been shown to decrease the amount of CME in patients with high-risk characteristics such as diabetes. Also in PXF, steroid and NSAID combination treatment has shown less inflammation and change in macular thickness when compared to steroid monotherapy. ${ }^{13}$ The combination treatment is believed to re-establish blood-aqueous barrier and thus reduce inflammation and PCME more efficiently than monotherapy with either drug alone. ${ }^{14}$ Interestingly, this combination treatment lacks evidence over NSAID monotherapy against PCME in high-risk patients.

Currently, no consensus exists on whether patients with PXF ought to be given systematically more aggressive postoperative anti-inflammatory medication and whether additional phase controls are warranted. Before introduction of new recommendations of anti-inflammatory medication on PXF, evaluation of the macular edema after cataract surgery and risk for PCME is warranted. Here, we analyzed central retinal thickness (CRT) change in eyes with PXF compared to those without after uncomplicated cataract surgery in patients treated either with steroid or with NSAID therapy.

\section{Materials and methods}

\section{Study design}

The study was a post-hoc analysis of a randomized, doubleblind, prospective single-center study. ${ }^{12}$ Patients were enrolled between January 2016 and October 2016 as per the national guidelines for the management of cataract in the Department of Ophthalmology, Kymenlaakso Central Hospital, Kotka, Finland. Postoperative preservative-free anti-inflammatory medication ( $\mathrm{N}=78$ eyes/study group) three times a day (t.i.d.) was prescribed for 3 weeks after cataract surgery to patients having either steroid (Monopex ${ }^{\circledR}$, dexamethasone phosphate $1 \mathrm{mg} / \mathrm{mL}$, Laboratoires Théa Clermont-Ferrand, France) or NSAID (Voltaren Ophtha ${ }^{\circledR}$, diclofenac sodium $1 \mathrm{mg} / \mathrm{mL}$, Laboratoires Théa) therapy. The presence of PXF was confirmed by the operating physician at preoperative evaluation immediately before surgery. The primary outcomes were anterior chamber flare at 28 days and change in CRT between measurements. Secondary outcomes were visual acuity gain and subjective irritation symptoms.

Inclusion criteria. The study subjects were of 60-90 years of age and were eligible for cataract surgery under the Current Care Guidelines of Cataract Surgery of the Finnish Medical Society, Duodecim (updated in 2013).

Exclusion criteria. Exclusion criteria for the study included prior or active wet age-related macular degeneration, retinal vein/artery occlusion, retinal detachment, retinal necrosis, vitritis/endophthalmitis, vitreous hemorrhage, retinal phlebitis or optic neuritis, previous intraocular procedures (including fundus laser photocoagulation), planned anti-vascular endothelial growth factor treatments, myopia above -6.0 diopters. Alcohol abuse, thyroid disease with abnormal thyroid-stimulating hormone (TSH) levels, continuous use of anti-inflammatory drugs, and sensitivity to any of the medications used during or after the operation. Criteria for exclusion were also intraoperative complications (such as iris prolapse, use of sutures, and posterior capsule tear), failure to attend the postoperative control visit at $28 \pm 2$ days, or failure to use the anti-inflammatory medication as prescribed.

Prior enrollment of the contralateral eye was not considered as exclusion criterion. However, (1) no simultaneous bilateral cataract surgeries were performed to study patients, (2) randomization of the treatment group was performed before every surgery independently from prior contralateral eye surgery, and (3) the minimum gap between the surgeries was 1 month giving sufficient time to recover from the first surgery.

The study was conducted according to the tenets of the Declaration of Helsinki and was approved by the Finnish Medicines Agency Fimea and the Institutional Review Board of Helsinki University Hospital (EudraCT Number: 2015-003296-30). 
Table I. Baseline variables according to the pseudoexfoliation syndrome (PXF).

\begin{tabular}{|c|c|c|c|}
\hline & PXF- $(n=124)$ & PXF $+(n=32)$ & $P$ \\
\hline Gender (M/F) n/\% & $56: 68(45: 55 \%)$ & $10: 22(31: 69 \%)$ & 0.156 \\
\hline Age (years) & $76.3 \pm 0.6(62-90)$ & $77.6 \pm 1.1(62-89)$ & 0.228 \\
\hline BCVA (Snellen decimals) & $0.34 \pm 0.01(C F-0.70)$ & $0.37 \pm 0.04(\mathrm{HM}-1.00)$ & 0.418 \\
\hline IOP (mmHg) & $15.6 \pm 0.4(7-32)$ & $18.3 \pm 0.8(12-32)$ & 0.002 \\
\hline Glaucoma medication n/\% & $3(2 \%)$ & $9(28 \%)$ & $<0.001$ \\
\hline CRT mean $(\mu \mathrm{m})$ & $274.4 \pm 2.8(|77-4| 5)$ & $268.5 \pm 5.0(227-36 I)$ & 0.263 \\
\hline CRT $\max (\mu \mathrm{m})$ & $323.1 \pm 2.7(266-476)$ & $317.8 \pm 5.2(264-405)$ & 0.440 \\
\hline AMD n/\% & 37 (30\%) & 9 (28\%) & 0.850 \\
\hline iERM (pucker) n/\% & II (9\%) & $2(6 \%)$ & 0.477 \\
\hline Operation time (min) & $19.2 \pm 0.92(5-70)$ & $19.0 \pm 2.35(6-75)$ & 0.396 \\
\hline Phaco energy (CDE) & $17.6 \pm 0.79(5.55-5 \mid .34)$ & $16.6 \pm 1.57(5.83-35.06)$ & 0.434 \\
\hline Pupil extension device $n / \%$ & II (9\%) & $3(9 \%)$ & 0.579 \\
\hline CTR n/\% & $2(2 \%)$ & I (3\%) & 0.500 \\
\hline
\end{tabular}

AMD: age-related macular degeneration; BCVA: best corrected visual acuity; CDE: cumulative dissipated energy; CF: counting finger; CRT: central retinal thickness; CTR: capsular tension ring; HM: hand movement; iERM: idiopathic epiretinal membrane; IOP: intraocular pressure.

Baseline variables regarding (I) patient, (2) ophthalmic, (3) posterior segment, and (4) surgical parameters. Data are given as mean ( \pm SEM) and range or absolute number and proportion. For two-group comparisons, qualitative data were analyzed with the two-factor $\chi^{2}$ test (or with Fisher's exact test when values in any of the cells of a contingency table were below 5) and ordinal measurement scale, and continuous variables with the nonparametric Mann-Whitney $U$ test.

Note: Bold values are statistically significant.

\section{Clinical evaluation}

The diagnosis of PXF was confirmed at the preoperative biomicroscopic examination based on the visualization at slit lamp of the deposition of distinctive fibrillary material in the anterior segment of the eye pathognomonic to PXF. Thirty-two of the 156 eyes $(21 \%)$ were recognized with PXF: 17 of the 78 eyes (22\%) in the dexamethasone group and 15 of the 78 eyes (19\%) in the diclofenac group.

The patients were examined by an ophthalmologist preoperatively on the operation day and they visited a research technician at 28 days ( \pm 2 days) postoperatively. On both visits, aqueous flare was recorded by a laser flare meter (FM-600; Kowa Company, Ltd., Nagoya, Japan) and CRT by a spectral domain optical coherence tomography (SD-OCT; Heidelberg Eye Explorer Version 1.9.10.0 and HRA/SPECTRALIS ${ }^{\circledR}$ Viewing Module Version 6.0.9.0, Heidelberg Engineering GmbH, Heidelberg, Germany). The mean of five reliable aqueous flare measurements was used in the analysis. Follow-up 30-frame SD-OCT scans were performed with AutoRescan ${ }^{\mathrm{TM}}$ software and preoperative CRT max values were compared to those obtained 28 days after surgery. There is no common definition for PCME. The diagnosis for PCME was based on clinical appearance together with typical OCT-findings taken at the 28-day control visit. ${ }^{15,16}$ Adverse effects of topical medication, subjective visual recovery, and overall satisfaction of participants was documented by the research technician.

Best corrected visual acuity (BCVA) was preoperatively evaluated by the referring ophthalmologist and postoperatively with an auto-refractometer (AR-1s, NIDEK Co. Ltd, Aichi, Japan) at standardized light conditions. Intraocular pressure (IOP) was measured by rebound tonometry $\left(\mathrm{iCare}^{\circledR}\right.$ tonometer, Revenio Group, Vantaa, Finland). The duration of operation, phaco energy (cumulative dissipated energy $(\mathrm{CDE})$ ), and the use of intraocular surgical aid (StabilEyes ${ }^{\circledR}$ capsular tension ring, Abbott Medical Optics Inc., Santa Ana, CA; $6.25 \mathrm{~mm}$ Malyugin Ring ${ }^{\circledR}$ pupil extension device, MicroSurgical Technology, Redmond, WA) were recorded.

\section{Statistical analyses}

Data are given as mean \pm standard error of the mean, except for the absolute numbers and proportions for the nominal scale. IBM SPSS Statistics 24 (SPSS Inc., Somers, NY) was used for statistical analysis. For two-group comparisons, qualitative data were analyzed with the two-factor $\chi^{2}$ test (or with Fisher's exact test when values in any of the cells of a contingency table were below 5) and ordinal measurement scale, and continuous variables with the nonparametric Mann-Whitney $U$ test. $P \leqslant 0.05$ was considered statistically significant.

\section{Results}

\section{Baseline variables}

156 eyes of 149 patients were screened for PXF before cataract surgery. Thirty-two eyes were diagnosed with PXF. The baseline variables regarding patient, ophthalmic, posterior segment and surgical characteristics were comparable between eyes with PXF and those without, except for IOP $(P=0.002$, Table 1) and glaucoma medication $(P<0.001$, Table 1$)$. 


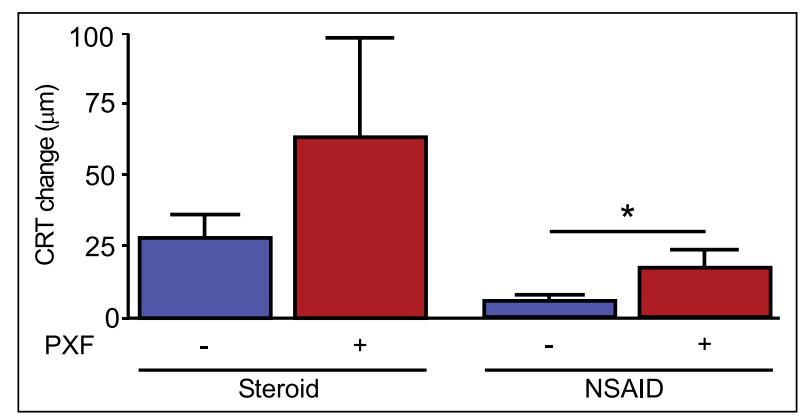

Figure I. Central retinal thickness in eyes with and without pseudoexfoliation. Data are given as mean \pm SEM. Change in CRT with steroid $(\mathrm{N}=78)$ or NSAID $(\mathrm{N}=78)$ monotherapy at 28 days after cataract surgery. CRT: central retinal thickness. $* P<0.05$.

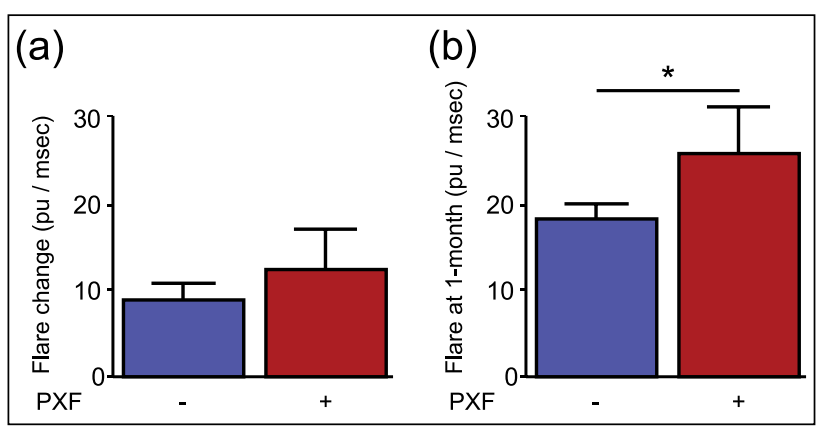

Figure 2. Aqueous flare in eyes with and without pseudoexfoliation. Data are given as mean \pm SEM. (a) Change in aqueous flare and (b) aqueous flare (pu/ms) at 28 days after cataract surgery.

PXF- $(\mathrm{N}=124) ; \mathrm{PXF}+(\mathrm{N}=32)$. $* P<0.05$.

\section{Aqueous flare and CRT in eyes with and without PXF}

In the dexamethasone treatment group, CRT increase was $63.3 \pm 35.5 \mu \mathrm{m}$ in eyes having PXF, and $28.9 \pm 8.0 \mu \mathrm{m}$ in eyes without $(P=0.975$, Figure 1$)$. In the diclofenac treatment group, CRT increase was $17.6 \pm 5.8 \mu \mathrm{m}$ in eyes with PXF, and $6.9 \pm 1.3 \mu \mathrm{m}$ in eyes without $(P=0.014$, Figure 1$)$.

At the day of surgery, aqueous flare was $11.4 \pm 1.3 \mathrm{pu} /$ $\mathrm{ms}$ in eyes with PXF and $8.9 \pm 0.7 \mathrm{pu} / \mathrm{ms}$ in eyes without $(P=0.019)$. Aqueous flare increase was $12.6 \pm 4.7 \mathrm{pu} / \mathrm{ms}$ in eyes with PXF and $9.2 \pm 1.8 \mathrm{pu} / \mathrm{ms}$ in eyes without $(P=0.096$, Figure 2(a)). At 28 days, aqueous flare was $25.8 \pm 5.4 \mathrm{pu} / \mathrm{ms}$ in eyes with PXF and $18.3 \pm 1.8 \mathrm{pu} / \mathrm{ms}$ in eyes without $(P=0.030$, Figure 2(b)).

In the dexamethasone treatment group, PCME was recognized in $18 \%$ ( 3 out of 17 ) of eyes with PXF and $7 \%$ (4 out of 61) of eyes without PXF $(P=0.171)$. In the diclofenac treatment group, PCME was recognized neither in PXF eyes, nor in eyes without PXF. According to the glaucoma medication, PCME was found in 1 of 12 eyes
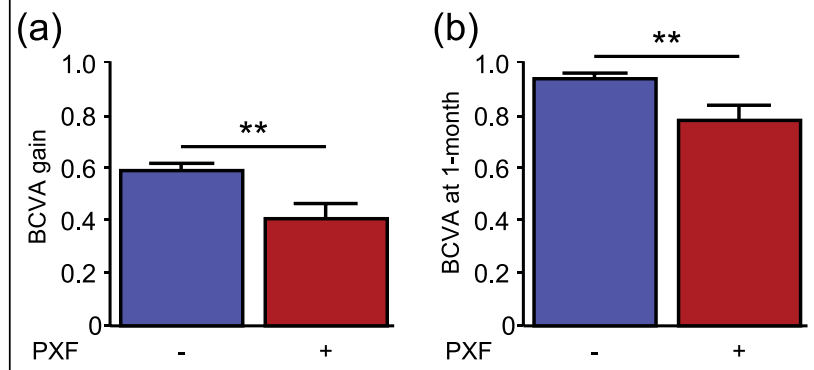

Figure 3. Postoperative BCVA in eyes with and without pseudoexfoliation. Data are given as mean \pm SEM. (a) BCVA gain and (b) BCVA at 28 days after cataract surgery. BCVA: best corrected visual acuity. PXF- $(\mathrm{N}=124) ; \mathrm{PXF}+(\mathrm{N}=32)$. $* * P<0.0$ I.

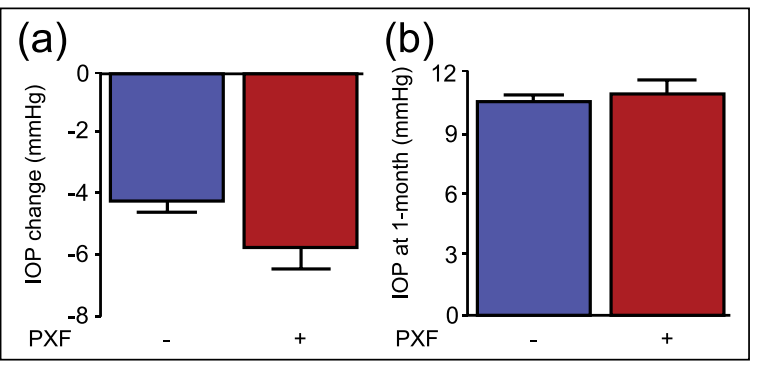

Figure 4. Intraocular pressure in eyes with and without pseudoexfoliation. Data are given as mean \pm SEM. (a) Change in IOP and (b) IOP at 28 days after cataract surgery.

IOP: intraocular pressure.

PXF- $(\mathrm{N}=124)$; $\mathrm{PXF}+(\mathrm{N}=32)$

(8\%) with glaucoma and 6 of 144 eyes (4\%) without glaucoma $(P=0.436)$.

\section{Visual acuity improvement and IOP in eyes with and without PXF}

BCVA gain was less in eyes with PXF compared to those without $(0.39 \pm 0.07$ vs $0.59 \pm 0.03$ decimals, $P=0.007$; Figure 3(a)). At 28 days, BCVA was impaired in eyes with PXF compared to those without $(0.77 \pm 0.06$ vs $0.92 \pm 0.03$ decimals, $P=0.008$, Figure 3(b)).

IOP decreased $7.1 \pm 0.9 \mathrm{mmHg}$ in eyes with PXF and $5.3 \pm 0.4 \mathrm{mmHg}$ in eyes without $(P=0.115$; Figure 4(a)). At 28 days, the IOP was $10.9 \pm 0.7 \mathrm{mmHg}$ in eyes with PXF and $10.5 \pm 0.3 \mathrm{mmHg}$ in eyes without $(P=0.541$; Figure 4(b)).

\section{Subjective irritation symptoms in eyes with and without PXF and patient satisfaction}

Subjective irritation symptoms from the topical medication were comparable between the eyes with PXF and those without. In the dexamethasone treatment group, 
subjective irritation in eyes with PXF and in those without was found in $12 \%$ ( 2 out of 17 ) and $8 \%$ (5 out of 61 ), respectively, whereas in the diclofenac treatment group, the respective values were $33 \%$ (5 out of 15 ) and 33\% (21 out of 63).

In the dexamethasone treatment group, in 24\% (4 out of 17) of eyes with PXF and $11 \%$ (7 out of 61 ) of eyes without PXF, patients reported that it had taken a week or more for visual acuity to rise to its eventual postoperative state $(P=0.242)$. In diclofenac treatment group, respective values were $6 \%$ ( 1 out of 17 ) and $8 \%$ ( 5 out of 61 ).

In the dexamethasone treatment group, a patient satisfaction survey (grade 1-10) revealed a satisfaction grade of $9.04 \pm 0.33$ in eyes with PXF and $9.24 \pm 0.16$ in eyes without PXF $(P=0.734)$. In the diclofenac treatment group, the respective satisfaction grades were $9.33 \pm 0.26$ and $9.50 \pm 0.10(P=0.677)$.

Interestingly, in PXF eyes with glaucoma $(N=9)$, none of the cases in the dexamethasone $(N=6)$ or in the diclofenac $(N=3)$ treatment group reported irritation symptoms from the anti-inflammatory drugs. Of note, baseline and postoperative aqueous flare and CRT were comparable in PXF eyes with and without glaucoma (data not shown).

\section{Discussion}

PCME is a common postoperative cause of visual loss after cataract surgery despite developed surgical techniques over the last few decades. ${ }^{17}$ Extensive studies on risk factors of PCME have been performed. Contralateral PCME, diabetic retinopathy, retinal vein occlusion, macular hole, and epiretinal membrane are related to the incidence of PCME. The role of prostaglandin (PG) analogs and other topical glaucoma medications affecting aqueous flare and PCME remains controversial. ${ }^{2,3,18-20}$

PXF eyes have been reported to have as high as 5- to 10 -fold increased risk for surgical complications. Various intra- and postoperative risks related to cataract surgery have been reported in PXF eyes, of which poor pupil dilation and zonular weakness have been identified as the most significant risk factors for surgical complications. Extensive blood-aqueous barrier breakdown in PXF eyes after cataract surgery leads to prolonged postoperative inflammation. Especially, eyes with glaucoma are expected to be at risk for PCME. ${ }^{21}$ Yet worth noticing is that PXF has not been verified as an independent risk factor for PCME. Our results emphasize that macular edema after cataract surgery is increased in eyes with PXF.

To prevent PCME, topical NSAIDs have been recommended after routine cataract surgery. ${ }^{11,12}$ Evidence-based guidelines are changing diverse patterns of postoperative management of cataract surgery. ${ }^{22}$ On the other hand, based on a systematic review, there is still uncertainty regarding topical NSAIDs in reducing the risk of poor visual outcome after cataract surgery. ${ }^{23}$ Interestingly, we previously found that short-term visual recovery was better in NSAID compared to steroid monotherapy. ${ }^{12}$ Our results support the recent findings of Coassin et al. ${ }^{13}$ proposing that topical steroid monotherapy provides incomplete anti-inflammatory effects in high-risk patients, including eyes with PXF. Whether steroid and NSAID combination therapy provides added benefit compared to NSAID monotherapy in high-risk patients remains to be solved. In our study, there was no difference in postoperative aqueous flare and CRT increase in PXF eyes with and without glaucoma. On the contrary, with a larger sample size, it was shown that the presence of glaucoma in PXF eyes may have a bearing on the foveal thickness increase, ${ }^{24}$ which should be taken into consideration in any study aiming to define a prophylactic treatment protocol for PCME.

Our 28-day follow-up was adopted from the Current Care Guidelines of Cataract Surgery of the Finnish Medical Society, Duodecim (updated in year 2013). As study limitation, we lack the long-term follow-up, 3-12 months after surgery. Studies with longer follow-up are needed to give even more thorough understanding to the anti-inflammatory effects of postoperative medications, prevention of PCME, and especially chronic macular edema leading to visual loss in high-risk patients.

Pseudophakic cystoid macular edema: update 2016 underlines the need to classify cataract patients as normal or high-risk patients and the importance of further studies considering anti-inflammatory medication to prevent PCME. ${ }^{3}$ Randomized clinical trials to define, for example, the optimal postoperative anti-inflammatory medication, timing, and drug delivery in various high-risk patients undergoing cataract surgery, including eyes with PXF, are warranted.

\section{Declaration of conflicting interests}

The author(s) declared no potential conflicts of interest with respect to the research, authorship, and/or publication of this article.

\section{Funding}

The author(s) disclosed receipt of the following financial support for the research, authorship, and/or publication of this article: The study was supported by grants from the Helsinki University Hospital Specific Catchment Area Clinical Research Grants (EVO116, EVO117, and EVO315), the Finnish Eye Foundation, Finnish Ophthalmological Society, the Nissi Foundation, Orion Research Foundation, Paulo Foundation and the Waldemar von Frenckell Foundation, Helsinki, Finland.

\section{References}

1. Kessel L. Can we meet the future demands for cataract surgery? Acta Ophthalmol 2011; 89: e289-e290.

2. Chu CJ, Johnston RL, Buscombe C, et al. Risk factors and incidence of macular edema after cataract surgery: a database study of 81984 eyes. Ophthalmology 2016; 123: 316-323.

3. Grzybowski A, Sikorski BL, Ascaso FJ, et al. Pseudophakic cystoid macular edema: update 2016. Clin Interv Aging 2016; 11: 1221-1229. 
4. Puska $P$ and Tarkkanen A. Exfoliation syndrome as a risk factor for cataract development: five-year follow-up of lens opacities in exfoliation syndrome. J Cataract Refr Surg 2001; 27: 1992-1998.

5. Ritch R and Schlotzer-Schrehardt U. Exfoliation syndrome. Surv Ophthalmol 2001; 45: 265-315.

6. Aboobakar IF, Johnson WM, Stamer WD, et al. Major review: exfoliation syndrome; advances in disease genetics, molecular biology, and epidemiology. Exp Eye Res 2017; 154: 88-103.

7. Aung T, Ozaki M, Lee MC, et al. Genetic association study of exfoliation syndrome identifies a protective rare variant at LOXL1 and five new susceptibility loci. Nat Genet 2017; 49: 993-1004.

8. Krause U, Alanko HI, Karna J, et al. Prevalence of exfoliation syndrome in Finland. Acta Ophthalmol Suppl 1988; 184: $120-122$.

9. Kuchle M, Nguyen NX, Hannappel E, et al. The bloodaqueous barrier in eyes with pseudoexfoliation syndrome. Ophthalmic Res 1995; 27(Suppl. 1): 136-142.

10. Schumacher S, Nguyen NX, Kuchle M, et al. Quantification of aqueous flare after phacoemulsification with intraocular lens implantation in eyes with pseudoexfoliation syndrome. Arch Ophthalmol: Chic 1999; 117: 733-735.

11. Kessel L, Tendal B, Jorgensen KJ, et al. Post-cataract prevention of inflammation and macular edema by steroid and nonsteroidal anti-inflammatory eye drops: a systematic review. Ophthalmology 2014; 121: 1915-1924.

12. Ylinen PHE, Laine I, Lindholm JM, et al. Anti-inflammatory medication following cataract surgery: a randomized trial between preservative-free dexamethasone, diclofenac and their combination. Acta Ophthalmol. Epub ahead of print 25 January 2018. DOI: 10.1111/aos.13670.

13. Coassin M, Iovieno A, Soldani A, et al. Bromfenac ophthalmic solution $0.09 \%$ as an adjunctive therapy to topical steroids after cataract surgery in pseudoexfoliation syndrome. $J$ Cataract Refr Surg 2016; 42: 1119-1125.

14. Kim SJ, Flach AJ and Jampol LM. Nonsteroidal antiinflammatory drugs in ophthalmology. Surv Ophthalmol 2010; 55: 108-133.
15. Antcliff RJ, Stanford MR, Chauhan DS, et al. Comparison between optical coherence tomography and fundus fluorescein angiography for the detection of cystoid macular edema in patients with uveitis. Ophthalmology 2000; 107: 593-599.

16. Kusbeci T, Eryigit L, Yavas G, et al. Evaluation of cystoid macular edema using optical coherence tomography and fundus fluorescein angiography after uncomplicated phacoemulsification surgery. Curr Eye Res 2012; 37: 327-333.

17. Hernstadt DJ and Husain R. Effect of prostaglandin analogue use on the development of cystoid macular edema after phacoemulsification using STROBE statement methodology. J Cataract Refr Surg 2017; 43: 564-569.

18. McCafferty S, Harris A, Kew C, et al. Pseudophakic cystoid macular edema prevention and risk factors; prospective study with adjunctive once daily topical nepafenac $0.3 \%$ versus placebo. BMC Ophthalmol 2017; 17: 16.

19. Lee KM, Lee EJ, Kim TW, et al. Pseudophakic macular edema in primary open-angle glaucoma: a prospective study using spectral-domain optical coherence tomography. Am J Ophthalmol 2017; 179: 97-109.

20. Wendel C, Zakrzewski H, Carleton B, et al. Association of post-operative topical prostaglandin analog or beta-blocker use and incidence of pseudophakic cystoid macular edema. J Glaucoma 2018; 27(5): 402-406.

21. Shingleton BJ, Crandall AS and Ahmed II. Pseudoexfoliation and the cataract surgeon: preoperative, intraoperative, and postoperative issues related to intraocular pressure, cataract, and intraocular lenses. J Cataract Refr Surg 2009; 35: 1101-1120.

22. Kessel L, Erngaard D, Flesner P, et al. Do evidencebased guidelines change clinical practice patterns? Acta Ophthalmol 2017; 95: 337-343.

23. Lim BX, Lim CH, Lim DK, et al. Prophylactic non-steroidal anti-inflammatory drugs for the prevention of macular oedema after cataract surgery. Cochrane Database Syst Rev 2016; 11: CD006683.

24. Yuksel N, Dogu B, Karabas VL, et al. Foveal thickness after phacoemulsification in patients with pseudoexfoliation syndrome, pseudoexfoliation glaucoma, or primary open-angle glaucoma. J Cataract Refr Surg 2008; 34: 1953-1957. 